Original Research Paper

\title{
Liquid Crystalline Polymers Compatibilization and Adhesion Enhancement by Reactive Blending in Post-Consumers PET's
}

\author{
Aversa Raffaella and Apicella Antonio \\ Advanced Materials Lab, Department of Architecture and Industrial Design, Second University of Naples, Aversa, Italy
}

Article history

Received: 07-06-2016

Revised: 09-06-2016

Accepted: 16-06-2016

Corresponding Author: Apicella Antonio

Advanced Materials Lab, Department of Architecture and Industrial Design, Second University of Naples, Aversa, Italy

Email: antonio.apicella@unina2.it

\begin{abstract}
A method for PET mechanical properties enhancement by reactive blending with HBA/HNA Liquid Crystalline Polymers for in situ highly fibrillar composites preparation is presented. LCP/PET blends were reactive extruded in presence of Pyromellitic Di-Anhydride (PMDA) and then characterized by Differential Scanning Calorimetry, Thermally Stimulated Currents and tensile mechanical properties. The formation of specific macromolecular structures, where the PET, the LCP and the reactive additive are involved, has been hypothesized in the reactively extruded blends from TSC analysis evidences. The use of a reactive additive improved the matrix LCP compatibilization and adhesion as indicated by the SEM analysis and mechanical testing. Moderate amounts of LCP in the PET (0.5 and 5\%) and small amounts of thermo-active and reactive compatibilizer in the blend $(0.3 \%)$ were found to significantly improve LCP melt dispersion, melts shear transfer and LCP fibril formation and adhesion. Blends of PET and LCP containing the compatibilizer favored the formation of a well dispersed and homogeneous fibrillar phase whose particle size distribution did not show great coarsening and coalescence leading to significant elastic properties improvements from 0.8 for not compatibilized to $3.1 \mathrm{GPa}$ for compatibilized $0.5 \%$ LCP loaded PET blends that was even higher than those expected from ordinary theoretical calculation. This unexpected improvement was probably due to the presence of two distinct phases' supra-molecular structures involving PET-LCP and PMDA.
\end{abstract}

Keywords: LCP, PET, Reactive Compatibilizer, Fibrillar in Situ Composites, Recycle

\section{Introduction}

Post-consumer PET is principally coming from the recover of injection blow moulded bottles prepared using high intrinsic viscosity and molecular weight solid state upgraded fibre grade PET's. Intrinsic Viscosity (IV) is a measure of the polymers Molecular Weight (MW) and therefore reflects the material's melting point, crystallinity and tensile strength. The polymer chain length in PET determines the molecular weight of the material and the mechanical properties that make the high IV PET useful for high strength applications. The higher the tensile, impact or operational temperature, the higher the polymer intrinsic viscosity should be. Applications of these high molecular weight and intrinsic viscosity PET's include carbonated beverage bottles, strapping tape, photographic film and tapes while amorphous state low IV forms are used in the fibre and textile industries.

However, post consumers high molecular weight PET suffers of a significant downgrading during the recycling process, which follows molecular weight decrease due to spontaneous hydrolysis processes in the molten state, that strongly reduces its intrinsic viscosity and the material final mechanical properties. These recycled materials could then be used only for low value and less structural applications such as fibers for carpets, fiber fill, geotextiles or extruded into sheet for thermo-forming and stretch blow molding of non-food containers.

Blending with glass or carbon fibers could help in improving the mechanical and thermal stability of such materials. However, processing of these composites not 
always results in the expected mechanical properties improvements (Apicella et al., 1980; Nicolais et al., 1981). Fibers breaking and high viscosity of the molten composite material during the process, strongly limit their application. Liquid crystalline polymers could exhibit very high potential as high strength modifiers that can be easily processed since tend to dissolve in the matrix at the high processing temperatures, reducing the melt viscosity and facilitating the extrusion and injection processes. Pure LCP (not in blend with a polymeric matrix) can be potentially used in several industrial fields such as electronics, composites and packaging where high dimensional stability, low aging and environment sensitivity are needed. However, once oriented, their highly anisotropic structure, while increasing its strength in the direction of orientation, strongly reduces their strength in the transverse direction. Blending with other engineering thermoplastics is therefore needed. Nevertheless, the morphology of such blends is unstable; the dispersed phase distribution and size, in fact, tend to coarsen when the melt is subjected to low shear conditions, e.g., as it may occur during certain injection molding conditions, leading to deleterious effects on the final blend properties.

The shear viscosity of the isotropic phase at high temperatures is, in fact, higher than the viscosity of the nematic phase at the lower temperatures. During flow, the mesophase domains easily slides on each other when the anisotropic phase is oriented along the flow direction, due to the fact that the LCPs possess relaxation times four times longer than ordinary polymers, the elongational flow of an anisotropic melt containing domains of local orientation induces their stretching and alignment which is preserved upon cooling in to solid state. Some investigations (Dutta et al., 1996) have also shown that the potential level or LCP order near to unity (0.90) that can be reached for specifically imposed stretching on the neat LCP, is never approached when in blend with a host matrix; the actual level of order at the same apparent stretching reaches values of $0.4 / 0.5$. This can be related to poor interactions between the two phases present in the melt; however, the more the matrix filler/interactions is improved by chemical modification, the higher the improvement will result (Cruz and Son, 2015; Zhang et al., 2000).

The resulting so called in situ composites, formed by a polymeric matrix with liquid crystalline reinforcing phase has been extensively investigated in the past decades (Apicella et al., 1989; Nicodemo et al., 1981; Skovby et al., 1991; Zhang et al., 2000; Goh and Tan, 2012; Cruz and Son, 2015). Even in such cases, however, the properties of the resulting materials were lower than expected. This was primarily due to the poor control of the interaction and adhesion between the oriented LCP phases and host matrix. This lack of adhesion is related to the weak Van der Waals forces bridging the segments of the LCP and the hosting matrix.

In our investigation, melt phases interactions and solid phases adhesion between the two components have been improved and controlled by a reactive alloying processes where the LC polymeric molecules containing potentially reactive groups (such as $\mathrm{OH}, \mathrm{NH}$ and the like) are blended with PET based host polymers in presence of an anhydride specific reaction promoter. Extrusion, filming tests on commercially HBA/HNA LCP (VECTRA 950) were carried out with recycled PET to produce blends of in situ composite films that, after thermal stretching at an appropriate temperature, showed improved LCP fibre/host polymer adhesion and higher mechanical properties.

\section{Materials, Apparatus and Procedures}

\section{Materials}

LCP fillers and reactive additives: Vectra A 950 (Hoechst) prepared from HBA and HNA and Pyromellitic Anhydride (PMDA) has been used as reactive additive.

Matrix: Recycled PET's of IV-0.60 dl/g have been used. Intrinsic viscosity has been measured at $25^{\circ} \mathrm{C}$ in phenolortodichlorobenzene (60/40 weight fraction) solutions.

\section{Apparatus and Procedures}

\section{Blending and Reactive Extrusion}

The PET matrix, the dispersed LCP and the reactive additive (PMDA) compounds were plasticated after previous drying $\left(24 \mathrm{~h}\right.$ at $140^{\circ} \mathrm{C}$ under vacuum) in a Haake counter rotating intermeshing twin screw extruder. Mixtures containing 0.5 and $5.0 \%$ of LCP with and without the reactive additive $(0.30 \%)$ were extruded at $290^{\circ} \mathrm{C}$, quenched in a water bath and then pelletized.

\section{Filming and Orientation}

Base polymer and mixtures in the form of pellets were dried in a vacuum oven at $140^{\circ} \mathrm{C}$ for $16 \mathrm{~h}$ and then extruder in a single screw extruder Haake equipped with a flat die. The strand of the blends and neat resin were extruded into a water bath and then calendered without stretching. General purpose tensile tester (Instron model 4500) equipped with a thermostatic chamber set at $190^{\circ} \mathrm{C}$ has been used to stretch under nitrogen the extruded strands up to two times the initial length at the crosshead speed of $1 \mathrm{~mm} / \mathrm{min}$.

\section{Mechanical Testing}

The tensile modulus of the stretched samples was measured on the same Instron 4500 mechanical tester with a constant crosshead speed of $10 \mathrm{~mm} / \mathrm{min}$ on $3 \mathrm{~cm}$ samples. 


\section{Differential Scanning Calorimetry}

An ADSC Mettler Differential Scanning Calorimeter has been used for the thermocalorimetric characterization. Samples films of $0.5 \mathrm{~mm}$ containing 0.5 and $5 \%$ of LCP before and after stretching and annealing were tested. Thermal scans from 0 to $300^{\circ} \mathrm{C}$ were carried out at $5^{\circ} \mathrm{C} / \mathrm{min}$.

\section{Thermally Stimulated Depolarization Currents (TSDC)}

A Solomat model 41000 spectrometer equipped with liquid nitrogen cooling system has been used on samples films of $0.5 \mathrm{~mm}$ containing $0.5 \%$ of LCP before and after stretching and annealing were tested. Thermal scans from 0 to $200^{\circ} \mathrm{C}$ were carried out were carried out at $5^{\circ} \mathrm{C} / \mathrm{min}$ on samples polarized for $5 \mathrm{~min}$ at 40,60 and $90^{\circ} \mathrm{C}$ in a polar field of $200 \mathrm{~V} / \mathrm{mm}$ and quenched to $-100^{\circ} \mathrm{C}$.

\section{Scanning Electron Microscopy (SEM)}

A Hitachi electron microscope has been used for surface morphology characterization. Namely, samples containing 0.5 and $5 \%$ of LCP were both fractured in brittle mode under nitrogen and in plastic mode in tensile tests. Fracture surfaces of the samples of extruded and extruded/stretched films were observed at 400 to $6000 \mathrm{X}$ magnification.

\section{Results and Discussion}

Reactive extrusion was carried out on polymer blends of the PET and the LCP previously mixed in a twin extruder by adding the $0.3 \%$ of the poly-anhydride (PMDA). The resulting material was extruded and films of different LCP content were obtained.

A preliminary thermo-calorimetric investigation on those samples indicated that for the compositions investigated this technique was not able to detect any difference between the samples with and without the reactive additive.

Figure 1 compares the thermogram relative to the PET/LCP (Vectra) blend with and without the reactive additive. Both curves show an inflection around $60^{\circ} \mathrm{C}$ that (for an oriented film) is representative of the glass transition temperature range of the polymer.

The intense peak at $250^{\circ} \mathrm{C}$ is relative to the melting of the crystalline phase.

The materials tested present crystallinity of about $50 \%$ taking the as reference heat of crystallization for the $100 \%$ crystalline PET $28.1 \mathrm{cal} / \mathrm{g}$ or $117 \mathrm{~J} / \mathrm{g}$ (Groeninckx et al., 1980).

In order to verify if the LCP and the host polymer underwent some chemical interaction during the reactive extrusion, a very sensitive analytical technique
(Teyssedre and Lacabanne, 1995) that measures the depolarization currents of thermally stimulated samples was used. Under an external electrical field the polymer (which is a dielectric medium) accumulates electric charges in a form that can be retained for long time. An increase of the internal segmental mobility can release these charges producing a depolarization current.

Peaks observed in the diagram reporting the intensity of such depolarization currents as a function of the temperature during a thermal scan are related to the release of electric charges in the samples undergoing physical events taking place at the segmental (molecular) and phase (super-molecular) level. Characteristic TSC diagrams of the blends containing $0.5 \%$ of the LCP (with and without PMDA) and of the pure LCP are compared in Fig. 2. This method provides high sensitivities even with currents of the order of 1013 Amps and lowers. The arrow on the curves indicates the temperature of polarization.

The diagram of the blend in absence of the reactive additive (PMDA) is characterized by a peak about $30^{\circ} \mathrm{C}$ above the polarization temperature that characterize the glass transition of the PET (around $60^{\circ} \mathrm{C}$ ) as well as that of the LCP (VECTRA) that, measured with the same technique (4), gave a sharper peak at $71.5^{\circ} \mathrm{C}$. The test performed in the same experimental conditions but on the same blend additivated with the reactive agent $(0.3 \%)$ is compared in the same Fig. 2 (darker curve).

Surprisingly, the new diagram does not present any peak in the region of the glass transition of the two blend components, moreover, a second thermal event is occurring around $110-140^{\circ} \mathrm{C}$ (which is evident as a broaden peak).

The TSC analysis, therefore, indicates that the presence of the reactive additive modifies the internal molecular structure of the blend. Even if in a very small amount, only $0.5 \%$, the LCP has been detected by the TSDC analysis. In the thermogram of the unreacted blend in Fig. 2, the peak is probably related to the presence of LCP while the presence of the peak at $140^{\circ} \mathrm{C}$ in the thermogram relative to the additivated blend is representative of a characteristic macromolecular structure where the PET, the LCP and the additive are involved. This second thermal event, which may be characteristic of this particular segmental rearrangement of the PET/LCP reacted blend, has been further confirmed in a TSDC test carried out on the additivated blend polarized at a higher temperature, namely $90^{\circ} \mathrm{C}$. At this high temperature, electric charges are accumulated and associated to events occurring at temperatures higher than $90^{\circ} \mathrm{C}$. By comparing this thermogram, Fig. 3, with that of the same sample polarized at the lower temperature, it is significantly evident this high temperature thermal event that is centered around $110-120^{\circ} \mathrm{C}$. 


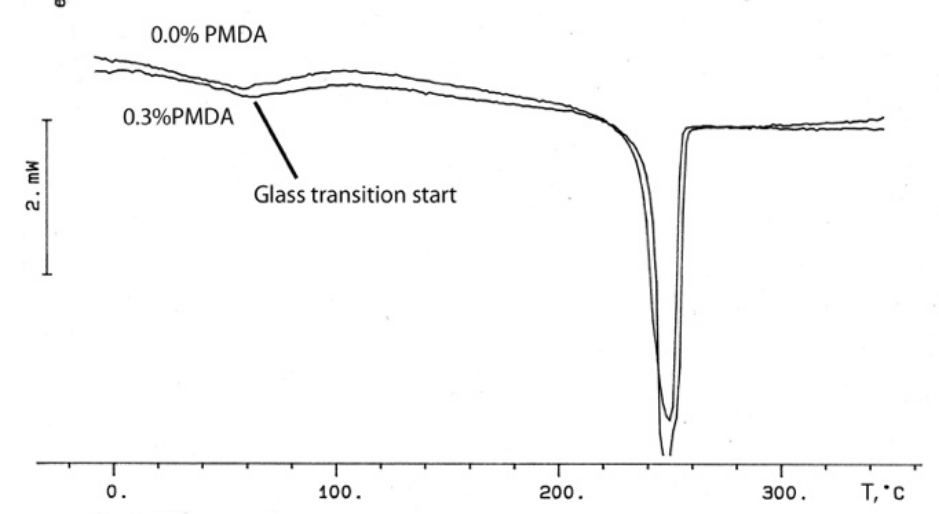

Fig. 1. DSC thermogram of PET/LCP oriented blends with and without reactive PMDA additive

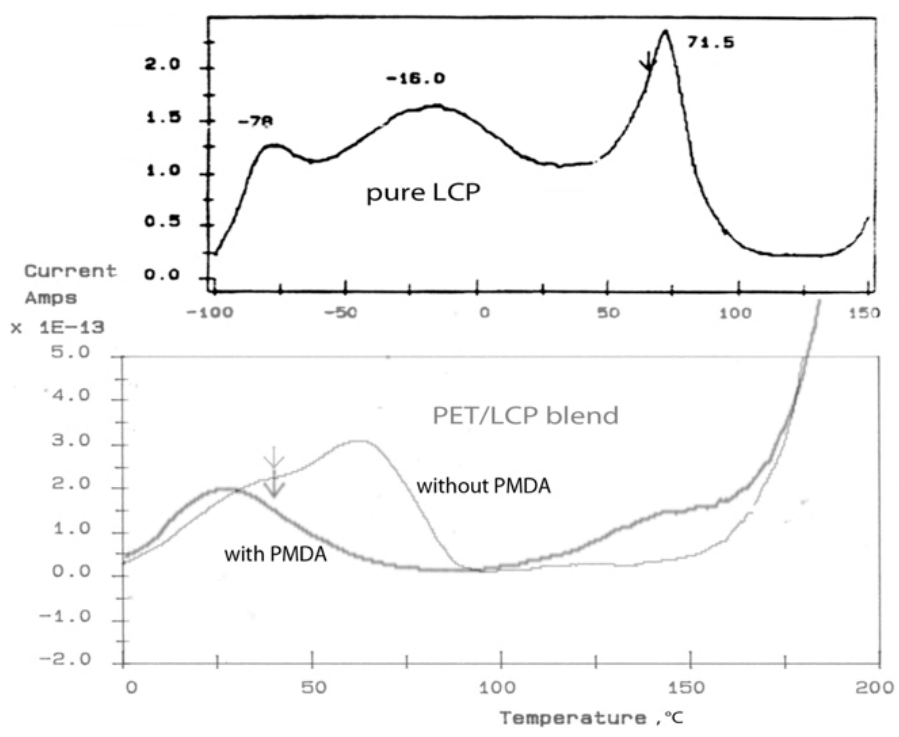

Fig. 2. Thermally Stimulated Current thermograms of PET/0,5\%LCP blends without (grey curve)and with PMDA reactive additive (black curve). Pure LCP on Top. Polarization $40^{\circ} \mathrm{C}$ for the blends and $60^{\circ} \mathrm{C}$ for the pure LCP

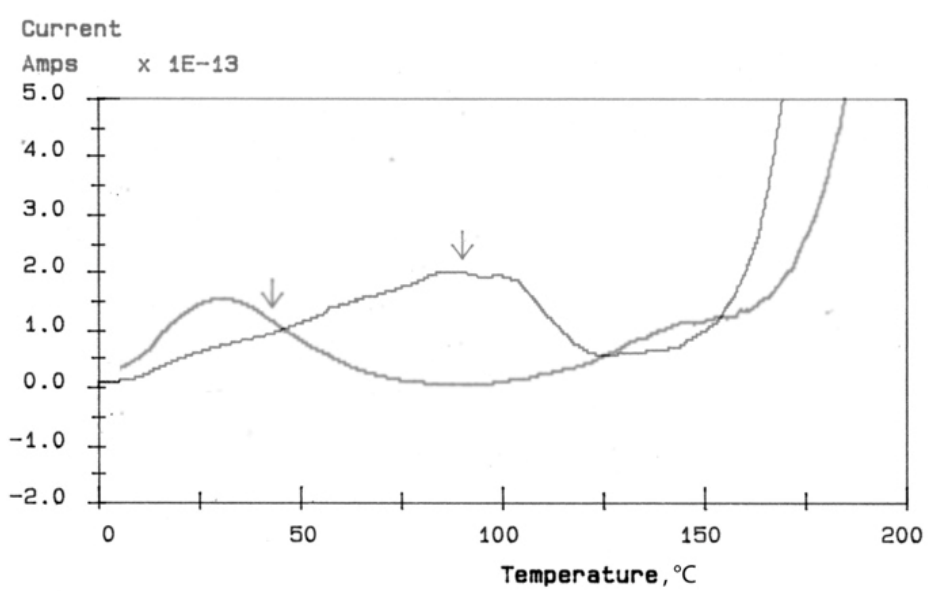

Fig. 3. Thermally Stimulated Current thermogram of PET/0,5\%LCP blends with $0,3 \%$ of PMDA reactive additive. Polarization 40 and $90^{\circ} \mathrm{C}$ 


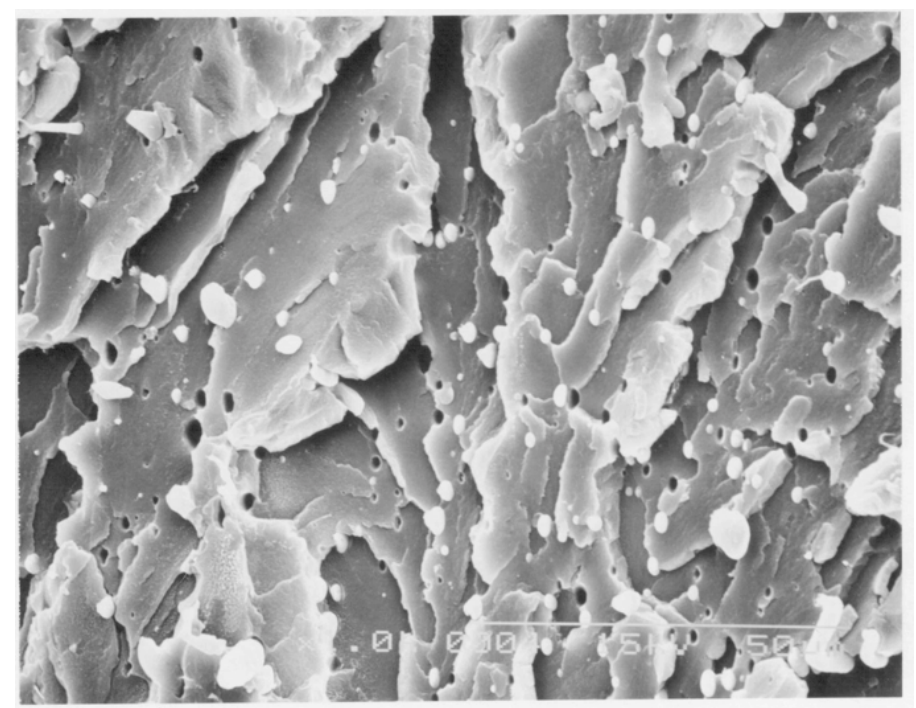

Fig. 4. SEM image of PET/5.0\%LCP blends without PMDA reactive additive

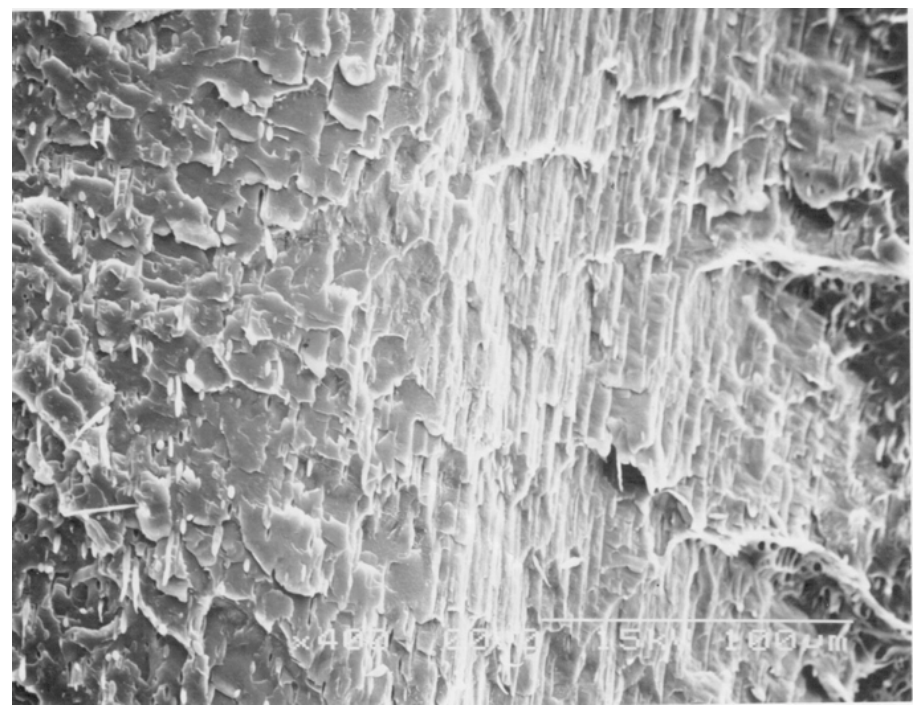

Fig. 5. SEM image of PET/5.0\%LCP blends with $0.3 \%$ of PMDA reactive additive

The thermal tests (DSC and TSDC) have been associated to a mechanical and Electron Scanning Microscopy (SEM) analyses.

The SEM analyses were run on PET containing 5\% of LCP fractured in liquid nitrogen.

Figure 4, shows the liquid crystalline polymer in the form of ellipsoid inclusion with low adhesion with the matrix (this hypothesis is validated by the presence of the spherical voids on the fracture surface that were presumably containing debonded LCP particles). The extrusion and filming procedures in absence of the reactive additive did not induce a significant orientation of the LCP as indicated by the almost spherical shape of the inclusion.
Fracture surface of the sample of PET/5.0\%LCP blend containing the $0.3 \%$ of the reactive additive is shown in Fig. 5. The same extrusion and filming procedures used for the previously described sample produced, in this case, homogeneously distributed and elongated fibrils of LCP. Reactive alloying extrusion favors a better dispersion by improving the phase's interactions between the two molten components.

The SEM analysis were run on the surfaces of the same PET/LCP blend samples with and without the reactive additive that have been fractured in tensile tests run at room temperature. As evident from Fig. 6, the in situ composite do not containing the additive, is characterized by the presence of ellipsoid particles (about 
$8 \times 4$ microns) of the liquid crystalline phase, dramatically yields and plastically fails around the LCP inclusions.

The presence of the not adhering ellipsoid inclusions, which remain un-deformed after matrix fracture, leads to an intense stress concentration and plastic flow at the PET matrix/filler interface. Due to the lack of interaction during processing and of adhesion in the solid state, the LCP filler, therefore, does not act as a reinforcing agent.

In presence of the PMDA, the better dispersed fibrillar (about 2 microns in diameter) and well oriented LCP phase stretches and yields, as indicated in Fig. 7 and 8 , improving the matrix mechanical properties.
This occurrence was probably due to the fact that the ameliorated physical dispersion in presence of the reactive additive leads to higher fillers surface/volume ratios, playing a significant role in the fracture tests carried out at room temperature. Figure 8 is a higher magnification of the previous image showing as a LCP fibril adheres to the matrix and plastically fails. This is an indication of the active role of the additive in improving both processing and ultimate mechanical properties of the PET/LCP blends. This occurrence further confirms the macromolecular nature of the structural modification hypothesized from the analysis of the TSC diagrams.

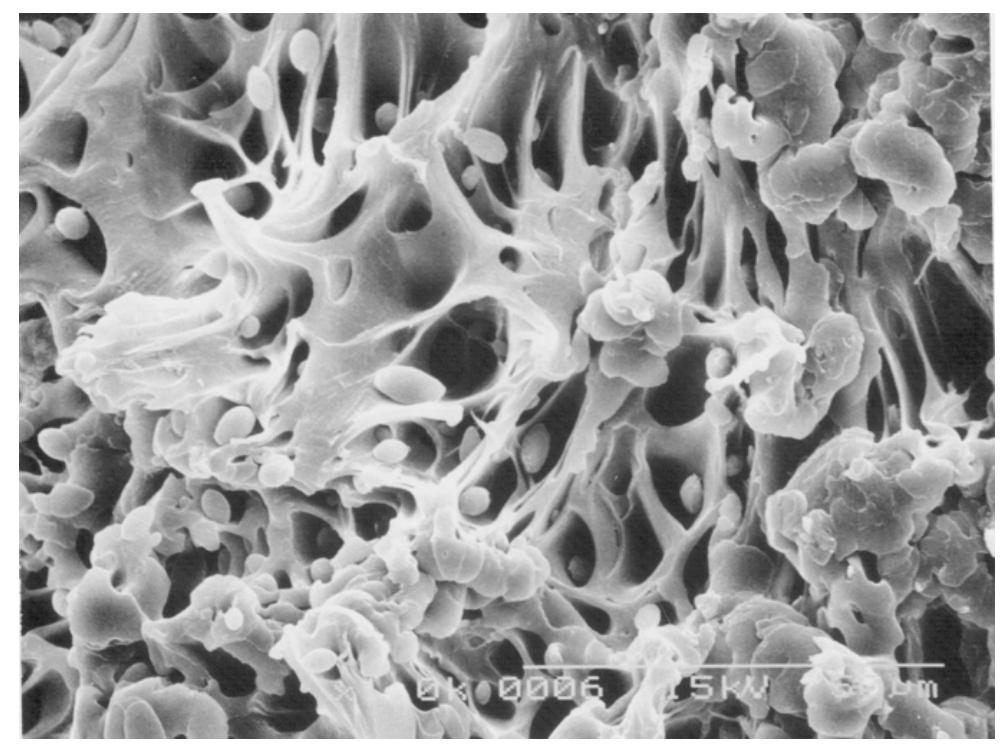

Fig. 6. SEM image of PET/5.0\%LCP blends without PMDA reactive additive. Room temperature fracture is accompanied by plastic flow around poor adhering LCP fillers

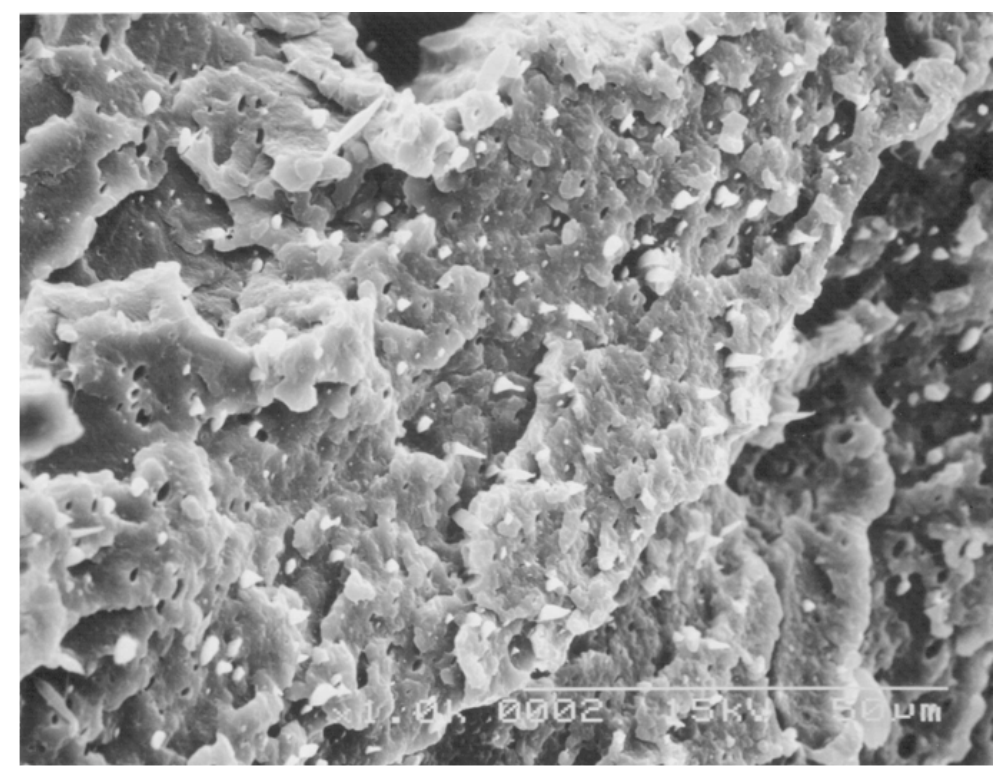

Fig. 7. SEM image of PET/5.0\%LCP blends with PMDA reactive additive. Good adhering and plastically deforming LCP fillers 


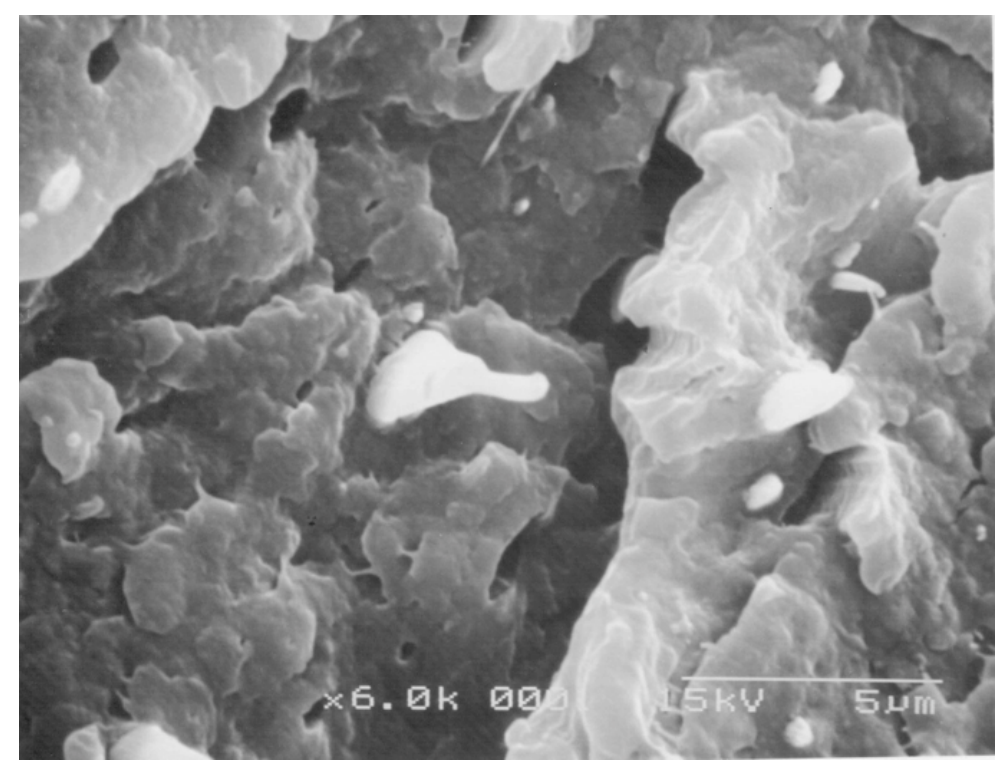

Fig. 8. Higher magnification of the previous SEM image showing the plastic deformation of the LCP filler

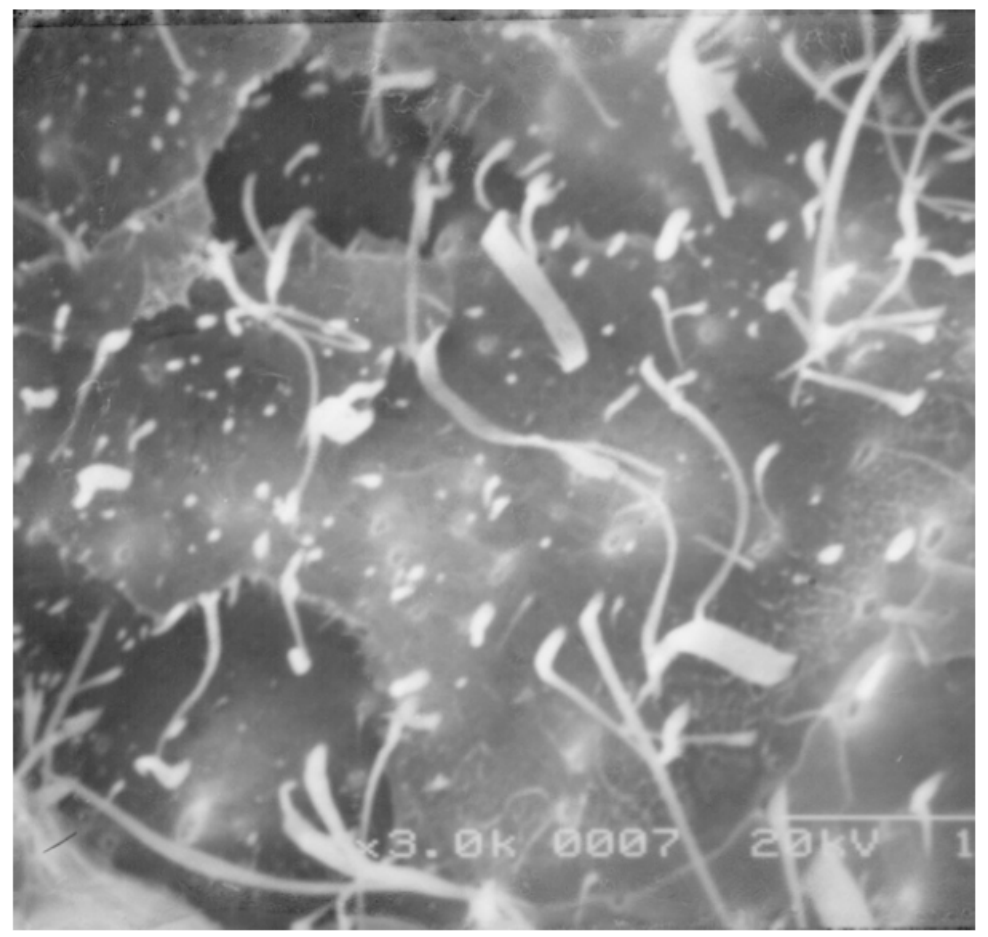

Fig. 9. High dispersion and yield of the LCP filler in the reactive additive compatibilized PET/LCP blends

\section{Mechanical Analysis}

As previously mentioned, the LCP in the extruded blend orients much less than the optically measured or SEM dimensional changes indicate, this may due to the lack of shear stresses transfer between LCP and matrix. The increases of elastic moduli calculated according the well accepted Halpin-Tsai relationships are, generally, much lower than expected: Values of $0.8 \mathrm{GPa}$ instead 1.0 $\mathrm{GPa}$ were in fact calculated). Nevertheless, the presence of the reactive compatibilizer favored both processes of shear transfer and post cure chemical bonding between LCP the host polymer.

In our investigation blend with 0.5 and $5 \%$ of LCP were utilized in presence and absence of specific reaction promoters. Moreover tests on the net resin in the 
same conditions of thermal stretching filming and processing were carried out as control.

Improvement of adhesion and mechanical properties in presence of the reaction promoters were always observed after stretching of the films (Fig. 9). Surprisingly the best result (even as absolute values) were observed for the blends of lower content of LCP $(0.5 \%)$ with increase of elastic moduli that were even twice higher than those expected from the mechanical H.T. theory (i.e., 3.9 GPa Vs the expected 2.1 GPa and the $2.0 \mathrm{GPa}$ in absence of LCP under the same overall processing conditions).

This unexpected high value could be due to higher level of LCP internal order and corresponding limiting elastic moduli near to the theoretical.

This was probably due to the fact that the ameliorated physical dispersion in presence of the reactive compatibilizer, which increases the number and dispersion of fibrils, plays a significant role in the LCP orientation even in the preliminary filming procedures.

For the as cast films, it was observed, in fact, that the presence of the reacting promoter produced films of higher moduli than the ordinary blending procedures: The expected moduli (according to Halpin Tsay theory) were only $10 \%$ lower expected for the activated $0.5 \%$ LCP blend and 50\% lower for the richer blend while the corresponding values in absence of the reacting promoter were respectively 30 and $80 \%$ lower.

Increase of the LCP orientation shown in Fig. 8 (or level of order) depends both on physical and chemical adhesion; a good physical dispersion during the extrusion leads to thinner fibers presenting a high surface to volume ratio. Good shear transfer will improve local LCP order and hence LCP elastic moduli. Moreover, additional chemical bonding under thermal stretching should further increase the mechanical properties of the LCP/Polymer blend.

\section{Conclusion}

The continuous increase in electronics components applications is expected to increase the liquid crystal polymer market growth over the next years. Increasing microinjection-molding demand coupled with high-temperature resistance and flow properties are expected to drive demand. Thin wall design, in fact, is critical during microinjection molding of electronic components. LCP is then preferred for manufacturing ultra-thin components up to $0.1 \mathrm{~mm}$ over other engineering resins such as Polyphenylene Sulfide (PPS), polycyclohexylenedimethylene terephthalate (PCT) and polyphthalamide (PPA) and nylons. Due to their higher processing viscosities (Son et al., 2004), these engineering resins take longer manufacturing cycles and can produce components restricted to $0.25 \mathrm{~mm}$ thickness.

Reactive blending of these engineering polymers with LCP's in presence of a reactive compatibilizer may result in both processing aids and mechanical enhancements (Weiss et al., 1987; Zhang et al., 2000).

Generally, in situ LCP composites are composed by an incompatible liquid crystalline polymer and by an ordinary thermoplastic polymer, which forms a molten matrix including and dispersing, as temperature of the melt is lowered, phase's separation in anisotropic and isotropic states. Upon further cooling and component solidification, the two phases structure is preserved. The resulting in situ composite presents a morphology that strongly depends on the two components rheological properties and on the processing conditions (Apicella et al., 1989). The liquid crystalline nematic anisotropic phase, in fact, easily aligns in the direction of the melt flow with a level of orientation that depends either upon the relative ratio between the two molten phases viscosities, the extensional character of the flow and the liquid filler dispersing ability of the molten polymeric matrix. In our research, as evident from TSC tests, the use of a reactive compatibilizer improved liquid phase interactions by a macromolecular structure creation involving PET, LCP and the reactive compatibilizer. The mechanical properties of the blends, which are defined by the resulting LCP/matrix morphology and content in the solid state, have been significantly improved by the presence of the reactive compatibilizer.

Independently on the anisotropic and isotropic character of the two polymer melts, a partial or total solubility of the LCPs in the host thermoplastic matrices in the molten state, however, cannot be excluded a priori. A liquid crystalline material is one that in addition to exhibiting an isotropic liquid phase also exhibits one or more anisotropic liquid phases; in the case of a high chemical affinity of the two blend components, the LCP can be partially or totally dissolved in the matrix. A plasticizing effect has been then (thermo calorimetrically) measured from the presence of the isotropic LCP melt which is dissolved in the matrix leading to a decrease of the blend melt viscosity and, hence, more easily processable in injection molded thin films. At elevated processing temperatures, the chemically similar to the PET host polymer LCP is probably completely dissolved in it further increasing the flexibility and softness of the melt by weakening the intermolecular bonding forces and the separation of host polymer chains. As cooling of the material proceeds, however, the LCP solubility could significantly decreases, finally leading to phase separation and LCP liquid crystallization in the still molten matrix.

Even if in some cases a complete miscibility it has been observed also in the final solid state, such is the case of Terephthalate Bis-4-n-Butylaniline (TBBA) in 
polystyrene (Apicella et al., 1986), the aromatic polyester LCPs based on p-Hydroxybenzoic Acid (HBA) and 2,6- Hydroxynaphtoic Acid (HNA) monomers used in our tests and for the recycled Poly-Ethylene Terephthalate (PET), an even limited partial solubility (Baird) occurred and it has been thermo-calorimetrically measured by Differential Scanning Calorimetry as PET glass transition lowering. This possibility, however, has been show from our tests to be enhanced by the presence of specific reactive additives.

The use of reactive blending agents (acting in such a case as reactive compatibilizer) and the choice of the proper LCP and host polymers (even if there is evidence that the blend is not extensively reacting in the preliminary short mixing and filming processing times) improves the level of dispersion and LCP orientation (especially for very low LCP contents).

The reason for the deviation from the law of mixtures is not completely clear. It is possible that for partially miscible systems, superior mechanical properties are obtained because the PET/LCP solid solution coexists with the free LCP phase. The matrix itself is reinforced to some extent by the LCP at a molecular structural level. Moreover, due to the partial miscibility, a higher interfacial adhesion between the PET/LCP solid solution and the free LCP phases strongly orient increasing the reinforcing effect. Another possibility is that because of the partial miscibility, there is lower interfacial surface tension, which leads to finer with higher aspect ratios providing more surface area and fibrils efficiency. Both mechanisms are probably occurring in our reactive blend system.

SEM of the fractured surfaces of the PET/LCP samples blended without PMDA (which has been hypothesized to be less miscible) show large gap between pulled out spheroidal inclusions and PET matrix and these voids reveal poor matrix/fibrils interfacial adhesion and higher matrix melt interfacial surface tension. The higher aspect ratio of the fibrils present in the PET/LCP systems blended in presence of PMDA indicates the possibility of higher matrix/filler interactions and lower matrix interfacial surface tension which favored LCP better dispersion.

The influence of LCP level of dispersion, orientation and thermally activated reaction under stretching is under study to define their effect on the poor physical aging characteristics of amorphous PET. Moreover, an optimization between LCP and reaction promoter content, film process conditions and thermal stretching operation are also in progress.

\section{Author's Contributions}

Prof. Aversa Raffaella, run all characterization tests and data interpretation. Prof. Apicella investigated on the base theoretical aspects.

\section{Ethics}

No ethical issues arise from this paper.

\section{References}

Apicella, A., L. Nicodemo and L. Nicolais, 1980. Recoil kinetics of uniaxially oriented polystyrene. Rheological Acta 19: 291-298.

DOI: $10.1007 / \mathrm{BF} 01543141$

Apicella, A., P. Iannelli, L. Nicodemo, L. Nicolais and A. Roviello et al., 1986. Dimensional stability of polystyrene-polymeric liquid crystal blends. Polymer Eng. And Sci., 26: 600-604.

DOI: $10.1002 /$ pen.760260904

Apicella, A., L. Nicodemo, L. Nicolais and R.A. Weiss, 1989. Dimensional stability of thermoplasticpolymeric liquid crystal blends. Polymer Sci. Technol.

Cruz, H. and Y. Son, 2015. Reactive compatibilization of liquid crystalline polymer/ethylene-acrylic acid ionomer blends. J. Korea Academia-Industrial Cooperat. Society, 16: 3653-3659. DOI: $10.5762 /$ KAIS.2015.16.5.3653

Dutta, D., R.A. Weiss and J. He, 1996. Compatibilization of blends containing thermotropic liquid crystalline polymers with sulfonateionomers. Polymer, 37: 429-435. DOI: 10.1016/0032-3861(96)82912-0

Goh, K.L. and L.P. Tan, 2012. Micromechanical fibrerecruitment model of liquid crystalline polymer reinforcing polycarbonate composites. Damage Fract. Comp. Mater. Struct., 17: 85-106. DOI: 10.1007/978-3-642-23659-4_7

Groeninckx, G., H. Reynaers, H. Berghmanz and G. Smets, 1980. Morphology and melting behavior of semicrystalline poly(ethylene terephthalate). I. Isothermally crystallized PET. J. Polymer Scie., 18: 1331-1324.

DOI: $10.1002 /$ pol.1980.180180612

Nicodemo, L., A, Apicella and L. Nicolais, 1981. Dimensional stability and tensile properties of drawn polystyrene composites. J. Applied Polymer Sci., 26: 129-139.

DOI: 10.1002/app.1981.070260111

Nicolais, L., A. Apicella and L. Nicodemo, 1981. Filler effect on the recoil kinetics of drawn polystyrene. Polymer Eng., 21: 151-154.

DOI: $10.1002 /$ pen.760210307

Son, Y., Y.S. Chun and R.A. Weiss, 2004. Improvement of the processability of poly (ether ketone ketone) by the addition of a liquid crystalline polymer. Polymer Eng. Sci., 44: 541-547. DOI: $10.1002 /$ pen.20048 
Skovby, M.H.B., J. Kops and R.A. Weiss, 1991. Studies on a diphenylether modified poly(phenyl-1,4, phenylene terephthalate) liquid crystalline polymer and its blends with polycarbonate and polysulfone. Polymer Eng. Sci., 31: 954-962. DOI: $10.1002 /$ pen. 760311305

Teyssedre, G. and C. Lacabanne, 1995. Some considerations about the analysis of thermo stimulated depolarization peaks. J. Phys. D, 28: 1478-1478. DOI: $10.1088 / 0022-3727 / 28 / 7 / 029$
Weiss, R.A., W. Huh and L. Nicolais, 1987. Novel reinforced polymers based on blends of polystyrene and a thermotropic liquid crystalline polymer. Polym. Eng. Sci., 27: 684-691. DOI: $10.1002 /$ pen. 760270913

Zhang, H., R.A. Weiss, J.E. Kuder and D. Cangiano, 2000. Reactive compatibilization of blends containing liquid crystalline polymers. Polymer, 41: 3069-3082. DOI: 10.1016/S0032-3861(99)00469-3 